\title{
A Simplified Approach to the Diagnosis of Atypical HUS: Clinical Considerations and Practical Implications
}

\author{
Arif Asif ${ }^{*}, 1$, Tushar Vachharajani ${ }^{2}$, Loay Salman ${ }^{3}$ and Ali Nayer ${ }^{3}$ \\ ${ }^{I}$ Division of Nephrology and Hypertension, Albany Medical College, Albany, NY, USA \\ ${ }^{2}$ Division of Nephrology and Hypertension W.G. (Bill) Hefner Veterans Affairs Medical Center, Salisbury, NC, USA \\ ${ }^{3}$ Division of Nephrology and Hypertension, University of Miami Miller School of Medicine, Miami, FL, USA
}

\begin{abstract}
Although rare, atypical hemolytic-uremic syndrome (aHUS) carries a high morbidity and mortality. Widespread microvascular thrombosis, thrombocytopenia and microangiopathic hemolytic anemia are the hallmark of aHUS. Virtually any organ (particularly the kidney) can be a target for the devastating effects of this syndrome. Uncontrolled activation of the alternative pathway of the complement system lies at the heart of the pathogenesis of aHUS. While significant advances have been made in our understanding of aHUS, establishing timely diagnosis of this syndrome has been challenging. This, in part, is due to the absence of a sensitive and specific diagnostic test and a relatively lack of our familiarity with the syndrome. With the recent success and approval of a humanized monoclonal antibody (eculizumab) in the treatment of aHUS, prompt and accurate diagnosis is of paramount importance to limit the target organ injury. This article presents a simplified approach to establishing the diagnosis of aHUS.
\end{abstract}

Keywords: Atypical hemolytic uremic syndrome, eculizumab, thrombotic microangiopathy, thrombotic thrombocytopenic purpura.

\section{INTRODUCTION}

Atypical hemolytic-uremic syndrome (aHUS) is a thrombotic microangiopathy (TMA). It is characterized by endothelial injury leading to vascular thrombosis [1-3]. Other thrombotic microangiopathies such as thrombotic thrombocytopenic purpura (TTP) and HUS associated with Shiga-toxin producing Escherichia coli (STEC-HUS) also cause vascular thrombosis. While all three diagnostic possibilities must be considered in a patient presenting with TMA, widespread familiarity with TTP (and its successful treatment with plasma therapy) and a conventional wisdom that aHUS is a disease of children lowers the index of suspicion of aHUS on the diagnostic list [3]. In this context, the diagnosis of aHUS can present a diagnostic challenge.

It is worth mentioning that a deficiency of the von Willebrand factor cleaving protease ADAMTS13 (a disintegrin and metalloproteinase with a thrombospondin type 1 motif, member 13) results in TTP. On the other hand, HUS has two different identifiable sources of origin [1-4]. The STEC-HUS is most often seen in the setting of an infection with Escherichia coli (usually E. coli serotypes 0157:H7 and 0104:H4). In contrast, uncontrolled activation of the alternative pathway of the complement system is involved in the pathogenesis of atypical HUS (aHUS) [3]. Of note, there is an intricate reciprocal interplay between complements and the coagulation system and complement activation facilitates vascular thrombosis [5]. In a

*Address correspondence to this author at the Division of Nephrology and Hypertension, Albany Medical College, 25 Hackett Blvd. Albany, NY 12208, USA; Tel: 518-262-0769; E-mail: AsifA@mail.amc.edu retrospective autopsy-based study, Hosler et al. conducted a histopathological examination of 56 individuals who succumbed to HUS $(n=31)$ or TTP $(n=25)$ [6]. In individuals with TTP, platelet-rich thrombi were present in the heart, pancreas, kidney, adrenal gland and brain, in descending order of severity. In those with HUS, however, thrombi consisted predominantly of fibrin and red cells and were most abundant in the kidney followed by the pancreas $(19 \%)$, adrenal glands $(13 \%)$, brain $(6 \%)$ and heart $(3 \%)$. No distinction between "typical" and "atypical" HUS was made in this study, however.

From a simplistic standpoint, there are distinct laboratory tests that are available to make the diagnosis of TTP (ADAMTS13 activity assay) and STEC-HUS (PCR or culture-based examination of a stool sample). However, a single confirmatory diagnostic test for aHUS does not exist. Indeed, microangiopathic hemolytic anemia, thrombocytopenia and target organ injury, particularly kidney damage, are essential clinical features of aHUS.

Microvascular thrombosis in atypical hemolytic-uremic syndrome results in ischemic injury to target organs [5-13]. The kidney is the most common organ involved $[3,13]$. In this context, hematuria, proteinuria, hypertension, azotemia and volume overload can all be seen [3, 13]. Important diagnostic elements include thrombocytopenia (platelet count $<150,000$ / $\mathrm{mcL}$ or $25 \%$ decrease from baseline), microangiopathic hemolytic anemia (schistocytes on blood film, elevated lactate dehydrogenase, decreased haptoglobin, decreased hemoglobin) and target organ injury (elevated blood urea nitrogen and creatinine, abnormal liver function tests, elevated pancreatic enzyme levels, stroke, myocardial infarction etc.) [3] (Fig. 1). 


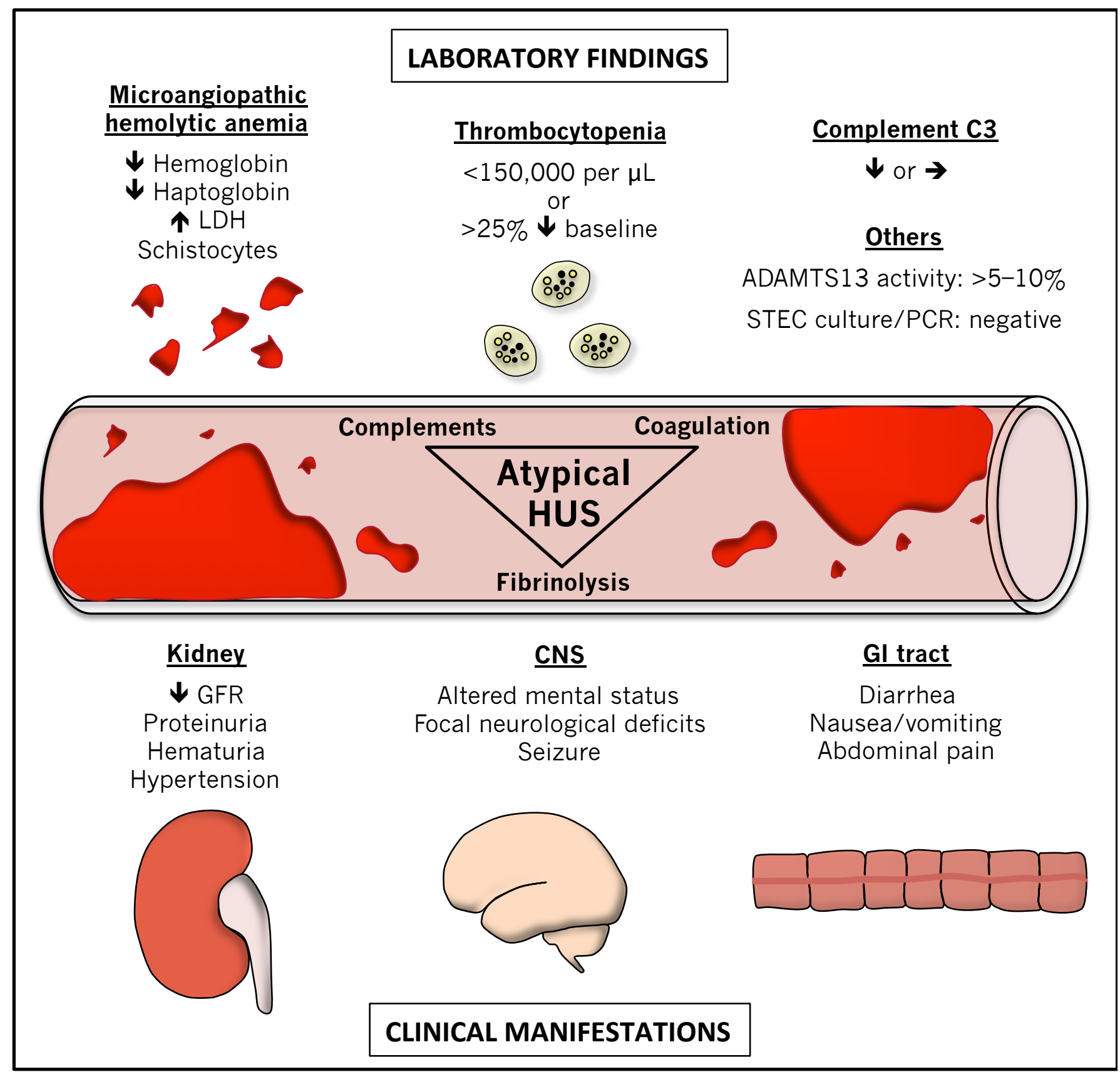

Fig. (1). Clinical and laboratory manifestations of atypical hemolytic-uremic syndrome. CNS, central nervous system; GFR, glomerular filtration rate; GI, gastrointestinal; LDH, lactate dehydrogenase; PCR, polymerase chain reaction; STEC, Shiga toxin-producing Escherichia coli; $\boldsymbol{\uparrow}$, increased; $\boldsymbol{\Downarrow}$, decreased; $\rightarrow$, unchanged; $>$, greater than; $<$, less than.

Laboratory investigations such as Shiga-toxin test and ADAMTS13 activity can be very helpful in distinguishing HUS, TTP and aHUS from one another. A deficiency of ADAMTS13 (less than $5 \%$ of normal activity) points to the diagnosis of TTP while the presence of Shiga toxin indicates STEC-HUS. Normal ADAMTS13 activity and absence of Shiga toxin help establish the diagnosis of aHUS in patients presenting with thrombotic microangiopathy. A major limitation of the ADAMTS13 activity assay test is the fact that the results may not be readily available and may take several days. In addition, the test must be performed prior to instituting any plasma therapy. Because TMA can cause rapid and progressive damage to vital organs relatively quickly, the luxury of waiting is not always the best approach $[3,13]$. In the past, clinicians have heavily relied on plasma therapy for the management of TMA. However, with the advent of eculizumab, a distinction between TTP and aHUS is vital earlier on. Awaiting ADAMTS13 results in the context of aHUS may render the patient vulnerable to the unchecked devastation of aHUS. Plasma therapy (which is often the first intervention) prescribed to TMA patients would not provide targeted and optimal results in patients with aHUS [8].

Recent data have emphasized that surrogate markers for severe ADAMTS13 deficiency can facilitate prompt 
Table 1. Serum creatinine level, platelet count and antinuclear antibodies in thrombotic thrombocytopenic purpura.

\begin{tabular}{|c|c|c|c|c|}
\hline Variable & Ferrari et al. $\mathrm{n}=35$ & Coppo et al.* $\mathrm{n}=160$ & Vesely et al. ${ }^{*} \mathrm{n}=18$ & Scully et al. $\mathrm{n}=80$ \\
\hline Serum creatinine, mean $(\mathrm{mg} / \mathrm{dL})$ & $1.26 \pm 0.59$ & $1.29 \pm 0.77$ & $1.8 \pm 1.2$ & $1.1(0.6-5.8)$ \\
\hline Serum creatinine $\geq 2.26 \mathrm{mg} / \mathrm{dL}$ & $8.6 \%$ & $\mathrm{~N} / \mathrm{A}$ & $16.7 \%$ & N/A \\
\hline Platelet count, mean $\left(\times 10^{9} / \mathrm{L}\right)$ & $17.4 \pm 13.7$ & $17.4 \pm 14.2$ & $11.6 \pm 6.9$ & $13.5(4-84)$ \\
\hline Platelet count $\geq 30\left(\times 10^{9} / \mathrm{L}\right)$ & $11.4 \%$ & N/A & $0 \%$ & $\mathrm{~N} / \mathrm{A}$ \\
\hline Antinuclear antibodies & N/A & $53 \%$ & N/A & $\mathrm{N} / \mathrm{A}$ \\
\hline
\end{tabular}

Data are expressed as mean \pm standard deviation or percentage. ${ }^{*}$ Patients with severe ADAMTS13 deficiency (less than $5 \%$ of normal activity). Numbers in parenthesis refer to the range of variable. N/A, not available.

diagnosis and may serve as a guide to treatment in patients presenting with a TMA [14-17]. It has been documented that a vast majority of patients with severe ADAMTS13 deficiency demonstrate a serum creatinine level less than $2.26 \mathrm{mg} / \mathrm{dL}$ and a platelet count less than $30 \times 10^{9} / \mathrm{L}$ (Table 1). In addition, Coppo et al. [15] reported antinuclear antibodies in $53 \%$ of patients with thrombotic microangiopathy associated with severe ADAMTS13 deficiency. A prediction model based on these three parameters indicates a specificity of $98.1 \%$ and a sensitivity of $46.9 \%$ for severe ADAMTS13 deficiency [15]. The sensitivity increases to $98.8 \%$ and the specificity decreases to $48.1 \%$ when only one criterion is present. Therefore, when thrombotic microangiopathy a associated with a serum creatinine level $\geq 2.26 \mathrm{mg} / \mathrm{dL}$ and a platelet count $\geq 30 \times 10^{9} / \mathrm{L}$, TTP is a highly unlikely diagnosis and atypical HUS should be strongly considered (Table 1) [14-17]. This approach can lead to early diagnosis of atypical HUS in the proper context and timely initiation of complement inhibition therapy using a humanized monoclonal antibody (eculizumab).

In general, Low $\mathrm{C} 3$ and normal $\mathrm{C} 4$ concentrations in the serum indicates the activation of the alternative pathway of the complement system with an understanding that decreased serum C3 level is not specific for aHUS [3]. Furthermore, the demonstration of normal $\mathrm{C} 3$ and $\mathrm{C} 4$ concentrations does not exclude the diagnosis of aHUS [3]. Finally, not all of the individuals who have an abnormality of complement proteins develop aHUS [3, 8]. Therefore, the demonstration of complement protein abnormalities is not required to make the diagnosis of aHUS $[3,10]$.

It should be noted that clinical presentations of TTP and HUS have challenged traditional wisdom [1, 3, 18-20]. In this context, TTP was thought to predominantly have neurological involvement. Nevertheless, altered mental status, focal neurological deficits and seizure have been reported in approximately $10 \%$ of patients with aHUS [8]. Moreover, nearly half of the children with aHUS are reported to have neurological involvement [18]. The presence of diarrhea has been thought to indicate STECHUS. However, nearly one third of the patients with aHUS present with diarrhea [19]. Once considered a disease of children, aHUS is increasingly diagnosed in the adult population [21, 22]. Consequently, conventional methodology of associating certain clinical features with TTP, STECHUS and aHUS is not an optimal approach.

It has been estimated that approximately half of the patients presenting with aHUS progress to end-stage renal disease within one year and a quarter of the patients die during the acute attack of the disease [1-4]. Even when transplanted, aHUS has a high rate of recurrence and subsequent damage to the transplanted organ and loss of graft [23-25]. Complement inhibition is emerging as an important strategy in stabilizing the platelet count, halting hemolysis and ameliorating renal injury [8, 13]. A humanized monoclonal antibody (eculizumab) has been successfully used for the management of aHUS [8]. In this context, establishing the diagnosis of aHUS in a timely fashion gains importance as eculizumab administration can directly limit the target organ damage. Prompt diagnosis is also important as another form of therapy (such as plasma exchange) is required for the treatment of TTP. At present, eculizumab is not indicated for the treatment of STEC-HUS.

\section{CONFLICT OF INTEREST}

The authors confirm that this article content has no conflict of interest.

\section{ACKNOWLEDGEMENTS}

This work received no funding from public, commercial or not-for-profit organizations.

\section{REFERENCES}

[1] Caprioli J, Noris M, Brioschi S, et al. Genetics of HUS: the impact of $\mathrm{MCP}, \mathrm{CFH}$, and IF mutations on clinical presentation, response to treatment, and outcome. Blood 2006; 108: 1267-79.

[2] Schmidtko J, Peine S, El-Housseini Y, Pascual M, Meier P. Treatment of atypical hemolytic uremic syndrome and thrombotic microangiopathies: a focus on eculizumab. Am J Kidney Dis 2013; 61: 289-99

[3] Laurence J. Atypical hemolytic uremic syndrome (aHUS): Making the diagnosis. Clin Adv Hematol Oncol 2012; 10: 3-9.

[4] Nayer A, Asif A: Atypical hemolytic uremic syndrome: a clinical review. Am J Ther 2014 (Eput ahead of print)

[5] Nayer A, Asif A. Atypical hemolytic-uremic syndrome: the interplay between complements and the coagulation system. Iran J Kidney Dis 2013; $7: 340-45$.

[6] Hosler GA, Cusumano AM, Hutchins GM. Thrombotic thrombocytopenic purpura and hemolytic uremic syndrome are distinct pathologic entities. A review of 56 autopsy cases. Arch Pathol Lab Med 2003; 127: 834-9.

[7] Goldberg RJ, Nakagawa T, Johnson RJ, Thurman JM. The role of endothelial cell injury in thrombotic microangiopathy. Am J Kidney Dis 2010; 56: 1168-74.

[8] Legendre CM, Licht C, Muus $\mathrm{P}$, et al. Terminal complement inhibitor eculizumab in atypical hemolytic-uremic syndrome. $\mathrm{N}$ Engl J Med 2013; 368: 2169-81.

[9] Loirat C, Frémeaux-Bacchi V. Atypical hemolytic uremic syndrome. Orphanet J Rare Dis 2011; 6: 1-30. 
[10] Noris M, Mescia F, Remuzzi G. STEC-HUS, atypical HUS and TTP are all diseases of complement activation. Nat Rev Nephrol 2012; 8: 622-33.

[11] Bomback AS, Smith RJ, Barile GR, et al. Eculizumab for dense deposit disease and C3 glomerulonephritis. Clin J Am Soc Nephrol 2012; 7: 748-56.

[12] Büttner-Mainik A, Parsons J, Jérôme $\mathrm{H}$, et al. Production of biologically active recombinant human factor $\mathrm{H}$ in Physcomitrella. Plant Biotechnol J 2011; 9: 373-83.

[13] Asif A, Haqqie SS, Ghate K, Mathew RO, Vachharajani T, Nayer A. Continued eculizumab therapy for persistent atypical hemolyticuremic syndrome. The Open Urol Nephrol J 2013; 6: 46-8.

[14] Ferrari S, Scheiflinger F, Rieger M, et al. French clinical and biological network on adult thrombotic microangiopathies. prognostic value of anti-ADAMTS 13 antibody features (Igisotype, titer, and inhibitory effect) in a cohort of 35 adult French patients undergoing a first episode of thrombotic microangiopathy with undetectable ADAMTS 13 activity. Blood 2007; 109: 2815-22.

[15] Coppo P, Schwarzinger M, Buffet M, et al. French reference center for thrombotic microangiopathies. predictive features of severe acquired ADAMTS13 deficiency in idiopathic thrombotic microangiopathies: the French TMA reference center experience. PLoS One 2010; 5: e10208.

[16] Vesely SK, George JN, Lämmle B, et al. ADAMTS13 activity in thrombotic thrombocytopenic purpura-hemolytic uremic syndrome: relation to presenting features and clinical outcomes in a prospective cohort of 142 patients. Blood 2003; 102: 60-8.
[17] Scully M, McDonald V, Cavenagh J, et al. A phase 2 study of the safety and efficacy of rituximab with plasma exchange in acute acquired thrombotic thrombocytopenic purpura. Blood 2011; 118: 1746-53.

[18] Neuhaus TJ, Calonder S, Leumann EP. Heterogeneity of atypical haemolyticuraemic syndromes. Arch Dis Child 1997; 76: 518-21.

[19] Zuber J, Le Quintrec M, Sberro-Soussan R, Loirat C, FrémeauxBacchi V, Legendre C. New insights into postrenal transplant hemolytic uremic syndrome. Nat Rev Nephrol 2011; 7: 23-35.

[20] Noris M, Brioschi S, Caprioli J, et al. International Registry of recurrent and familial HUS/TTP. familial haemolyticuraemic syndrome and an MCP mutation. Lancet 2003; 362: 1542-7.

[21] Noris M, Brioschi S, Caprioli J, et al. International registry of recurrent and familial HUS/TTP. familial haemolyticuraemic syndrome and an MCP mutation. Lancet 2003; 362: 1542-7.

[22] Bresin E, Rurali E, Caprioli J, et al. European working party on complement genetics in renal diseases. Combined complement gene mutations in atypical hemolytic uremic syndrome influence clinical phenotype. J Am Soc Nephrol 2013; 24: 475-86.

[23] Kavanagh D, Richards A, Goodship T, Jalanko H. Transplantation in atypical hemolytic uremic syndrome. Semin Thromb Hemost 2010; 36: 653-9.

[24] Lorcy N, Rioux-Leclercq N, Lombard ML, Le Pogamp P, Vigneau C. Three kidneys, two diseases, one antibody? Nephrol Dial Transplant 2011; 26: 3811-3.

[25] Waters AM, Licht C. aHUS caused by complement dysregulation: new therapies on the horizon. Pediatr Nephrol 2011; 26: 41-57.

Received: April 22, 2014

Revised: May 1, 2014

Accepted: May 6, 2014

(C) Asif et al.; Licensee Bentham Open.

This is an open access article licensed under the terms of the Creative Commons Attribution Non-Commercial License (http://creativecommons.org/licenses/by-nc/3.0/) which permits unrestricted, non-commercial use, distribution and reproduction in any medium, provided the work is properly cited. 